\title{
Novel TSPO-targeted Doxorubicin Prodrug for Colorectal Carcinoma Cells
}

\author{
JEMIANNE B. JIA ${ }^{1,2 *}$, XIAOXI LING ${ }^{3 *}$, MINZHI XING ${ }^{1,4}$, JOHANNES M. LUDWIG $^{1,4}$, \\ MINGFENG BAI ${ }^{5,6,7}$ and HYUN S. KIM ${ }^{1,4,7,8,9}$ \\ ${ }^{1}$ Interventional Oncology Translational Laboratory, \\ University of Pittsburgh School of Medicine, Pittsburgh, PA, U.S.A.; \\ ${ }^{2}$ Department of Radiology, Kaiser Permanente Los Angeles Medical Center, Los Angeles, CA, U.S.A.; \\ ${ }^{3}$ Department of Medicine, University of Pittsburgh School of Medicine, Pittsburgh, PA, U.S.A.; \\ ${ }^{4}$ Section of Interventional Radiology, Department of Radiology and Biomedical Imaging, \\ Yale School of Medicine, New Haven, CT, U.S.A.; \\ ${ }^{5}$ Department of Radiology, University of Pittsburgh School of Medicine, Pittsburgh, PA, U.S.A.; \\ ${ }^{6}$ Department of Bioengineering, University of Pittsburgh School of Medicine, Pittsburgh, PA, U.S.A.; \\ ${ }^{7}$ University of Pittsburgh Cancer Institute, Pittsburgh, PA, U.S.A.; \\ ${ }^{8}$ Section of Medical Oncology, Department of Medicine, Yale School of Medicine, New Haven, CT, U.S.A.; \\ ${ }^{9}$ Yale Cancer Center, Yale University, New Haven, CT, U.S.A.
}

\begin{abstract}
Background/Aim: $18 \mathrm{kDa}$ Translocator protein (TSPO) is a mitochondrial protein up-regulated in colorectal carcinoma $(C R C)$. Our purpose was to develop a TSPOtargeted doxorubicin prodrug (Dox-TSPO) which can be loaded onto drug-eluting beads for transarterial chemoembolization. Furthermore, we evaluated its loading and release kinetics and effects on cell viability. Materials and Methods: N-Fmoc-DOX14-O-hemiglutarate was coupled with a TSPO ligand, 6TSPOmbb732, using classical $N, N, N^{\prime}, N^{\prime}$-tetramethyl-O- $(1 \mathrm{H}$ benzotriazol-1-yl)uranium hexafluorophosphate coupling to produce Dox-TSPO. Loading and elution studies were performed using DC beads ${ }^{T M}$. Cell viability studies were performed using CellTiter-Glo ${ }^{\circledR}$ Luminescent Cell Viability
\end{abstract}

This article is freely accessible online.

*These Authors contributed equally to this study.

Correspondence to: Hyun S. Kim, MD, MHS, Section of Interventional Radiology, Department of Radiology and Biomedical Imaging, P.O. Box 208042, Yale School of Medicine, New Haven, CT 06510, U.S.A. Tel: +1 2037854747, Fax: +1 2037853024, email: kevin.kim@yale.edu and Mingfeng Bai, Ph.D., Vanderbilt University Institute of Imaging Science, 1161 21st Ave S AA-1105, Vanderbilt University School of Medicine, Nashville, TN 37232, U.S.A. Tel: +1 26153437753, Fax: +1 26153220734, e-mail: mingfeng.bai@vanderbilt.edu

Key Words: Targeted therapy, colorectal carcinoma, doxorubicin, TSPO, prodrug.
Assay. Results: Dox-TSPO was successfully synthesized and readily loaded onto and eluted from $D C$ beads ${ }^{T M}$, albeit at a slower rate than free doxorubicin. CRC cell lines expressing TSPO were 2- to 4-fold more sensitive to Dox-TSPO compared to free doxorubicin at $72 \mathrm{~h}$. Conclusion: Dox-TSPO is a promising candidate for targeted and directed cancer treatment of CRC liver metastases.

Colorectal cancer (CRC) is the third leading cause of cancer mortality in the US, with 50,000 deaths annually (1). CRC frequently metastasizes to the liver, with $15 \%$ of patients presenting with synchronous hepatic metastases and $29 \%$ developing metachronous metastases within 3 years of diagnosis (2). Hepatic metastases increase mortality. The 5year survival rate for patients with CRC if synchronous hepatic metastases are present is $2 \%$ compared to $90 \%$ if the cancer is localized to the colon (3).

The cytotoxic nature of systemic cancer treatments results in high rates of adverse effects making directed drug delivery systems an area of great interest. Transarterial chemoembolization (TACE) is a locoregional cancer treatment in which a chemotherapeutic agent and embolic material are directly injected into arteries supplying a tumor. This results in a dual method of tumor damage with higher concentrations of the chemotherapy in the area of the tumor than would be tolerated systemically and ischemia of the tissues secondary to embolization. A phase II study showed high response rates when colorectal hepatic metastases were treated with TACE using a combination of doxorubicin, mitomycin $\mathrm{C}$, and cisplatin (4). Drug-eluting bead TACE (DEB-TACE) is a variant in 
which micro-beads made from biocompatible polyvinyl alcohol saturated with chemotherapeutic agent are injected into the tumor vasculature. The use of micro-beads results in sustained release, increasing the time that tumor cells are exposed to the agent. The micro-beads are permanent and result in durable occlusion of arteries. DEB-TACE with irinotecan beads for colorectal metastases to the liver was demonstrated to improve overall and progression-free survival, and quality of life when compared to systemic chemotherapy in a phase III study (5). To further improve drug delivery to tumor cells, there has been increasing interest in targeted agents.

The $18-\mathrm{kDa}$ translocator protein (TSPO), previously known as the peripheral benzodiazepine receptor, is a transmembrane protein found in the mitochondrial membrane of cells throughout the body (6). It plays an important role in steroidogenesis through regulation of cholesterol translocation across the mitochondrial membrane, in cell proliferation by mediating translocation of pre-proteins needed for energy into mitochondria, and in apoptosis through regulation and maintenance of the transmembrane potential $(6,7)$. TSPO is up-regulated in brain, colorectal, breast, oral cavity, and prostate tumor cell lines (8-13). Due to the up-regulation of TSPO in a number of cancer cell lines, it is a potential target in directed oncological therapy.

Samuelson et al. experimented with targeting dendrimers using a TSPO ligand in C6 rat glioma cells and MDA-MB231 human breast cancer cells, both of which have high TSPO expression. The targeted dendrimers were successfully internalized into the cancer cells but remained extracellular in the control group (14). Wyatt et al. performed a similar study synthesizing a TSPO-targeted near-infrared probe and showed successful targeting of MDA-MB-231 breast cancer cells in an in vivo nude mouse model (15).

TSPO targeting has also been studied for drug delivery. Margiotta et al. synthesized a novel picoplatin analog conjugated with a TSPO ligand and tested the targeted chemotherapeutic agent on SF188 and SF126 human glioma cells and C6 and RG2 rat glioma cells. The glioma cells exposed to the compound had 10- to 100-fold improved uptake when compared to free cisplatin. The new compound also demonstrated selectivity with 10-fold less activity against ovarian cells, which have low TSPO expression (16). In addition, our group recently reported a TSPO-targeted photosensitizer, IR700DX-6T, which caused effective TSPO+ cancer cell death and TSPO+ tumor inhibition through photodynamic therapy (17).

The purpose of this study was to develop and evaluate a novel drug delivery system including a TSPO-targeted doxorubicin prodrug (Dox-TSPO) and DEB-TACE. Doxorubicin is an anthracycline antibiotic that is an effective antineoplastic agent in many types of malignancy and has been successfully used in DEB-TACE for CRC metastasis to the liver (18); however, it is known to cause dose-limiting cardiotoxicity along with severe nausea, vomiting, and alopecia when used systemically (19). Evaluation included study of loading and release kinetics in beads used in DEB-TACE and in vitro cell viability studies. Through TSPO-targeting and DEB-TACE delivery, we expect improved drug efficacy and reduced adverse effects.

\section{Materials and Methods}

Chemicals. $\quad \operatorname{Pd}\left[\mathrm{P}(t-\mathrm{Bu})_{3}\right]_{2}$, hexamethylenediamine, potassium hydroxide, cetyltrimethylammonium bromide, toluene, $N, N, N^{\prime}, N^{\prime}$ tetramethyl- $O$-( $1 H$-benzotriazol-1-yl)uranium hexafluorophosphate (HBTU), $N, N$-diisopropylethylamine (DIPEA), dimethylformamide (DMF), piperidine, and trifluoroacetic acid (TFA) were purchased from Sigma Aldrich (Saint Louis, MO, USA). Doxorubicin hydrochloride was purchased from A Chemtek, Inc (Worcester, MA, USA). $N$-(9-Fluorenylmethoxycarbonyloxy)succinimide (Fmoc-OSu) was obtained from Oakwood Chemical (Estill, SC, USA). Glutaric anhydride was purchased from Alfa Aesar (Tewksbury, MA, USA).

Prodrug synthesis. $\mathrm{N}$-(2-Bromo-5-methoxybenzyl)- $\mathrm{N}$-(5-fluoro-2 phenoxyphenyl)acetamide, the TSPO ligand, was prepared according to Bai et al. (20). $\mathrm{N}$-Fmoc-DOX-14-O-hemiglutarate, the doxorubicin prodrug precursor, was prepared according to Nagy et al. (21). Dox-TSPO was synthesized by coupling of $N$-Fmoc-DOX14-O-hemiglutarate and 6-TSPOmbb732 using classical HBTU coupling followed by fluorenylmethoxycarbonyl protecting group (Fmoc) deprotection with piperidine according to Figure 1 (detailed in Supplementary Material). The final chemical structure was fully characterized by proton nuclear magnetic resonance spectroscopy ( ${ }^{1} \mathrm{H}$ NMR), Carbon-13 nuclear magnetic resonance spectroscopy $\left({ }^{13} \mathrm{C} \mathrm{NMR}\right)$ and mass spectrometry (MS) analysis.

Loading doxorubicin and Dox-TSPO into beads. DC beads ${ }^{\mathrm{TM}}$ were purchased from BTG (London, UK). DC beads ${ }^{\mathrm{TM}}$ are biocompatible polyvinyl alcohol hydrogel microspheres that are chemically modified to allow for controlled loading and elution of chemotherapeutic drugs. They are used clinically for TACE procedures. A solution of DC beads ${ }^{\mathrm{TM}}(100-300 \mu \mathrm{m} ; 200 \mu \mathrm{l})$ were added to a cuvette that was maintained at $37^{\circ} \mathrm{C}$. Sodium phosphate solution was removed from the bead solution and the remaining beads were rinsed with $200 \mu \mathrm{l}$ of water three times before the cuvette was charged with $990 \mu \mathrm{l}$ of water. Doxorubicin or DoxTSPO solution $(10 \mu \mathrm{l})$ in dimethyl sulfoxide (DMSO; $17 \mathrm{mM}$ ) were added to the cuvette and thoroughly mixed. The absorption of the solution at $480 \mathrm{~nm}$ was recorded every 30 seconds for the first 200 minutes and every minute thereafter. These loading studies were repeated using larger DC beads ${ }^{\mathrm{TM}}(300-500 \mu \mathrm{m} ; 200 \mu \mathrm{l})$.

Releasing doxorubicin and Dox-TSPO from beads. After DC beads $^{\mathrm{TM}}$ were loaded with doxorubicin or Dox-TSPO, the excess loading solution was removed and the bead slurry was rinsed with $200 \mu \mathrm{l}$ of water three times before being diluted with $200 \mu \mathrm{l}$ of water. The loaded bead suspension $(100 \mu \mathrm{l})$ was transferred to a cuvette and the excess water was removed. Dulbecco's minimum essential cell medium (DMEM; $1 \mathrm{ml}$ ) from Sigma Aldrich was added to the cuvette to initiate the release. The cuvette was incubated at $37^{\circ} \mathrm{C}$ for 7 days. The absorption of the solution at 480 $\mathrm{nm}$ was recorded every day. This was performed for solutions of both the $100-300 \mu \mathrm{m}$ and $300-500 \mu \mathrm{m}$ DC beads ${ }^{\mathrm{TM}}$. 

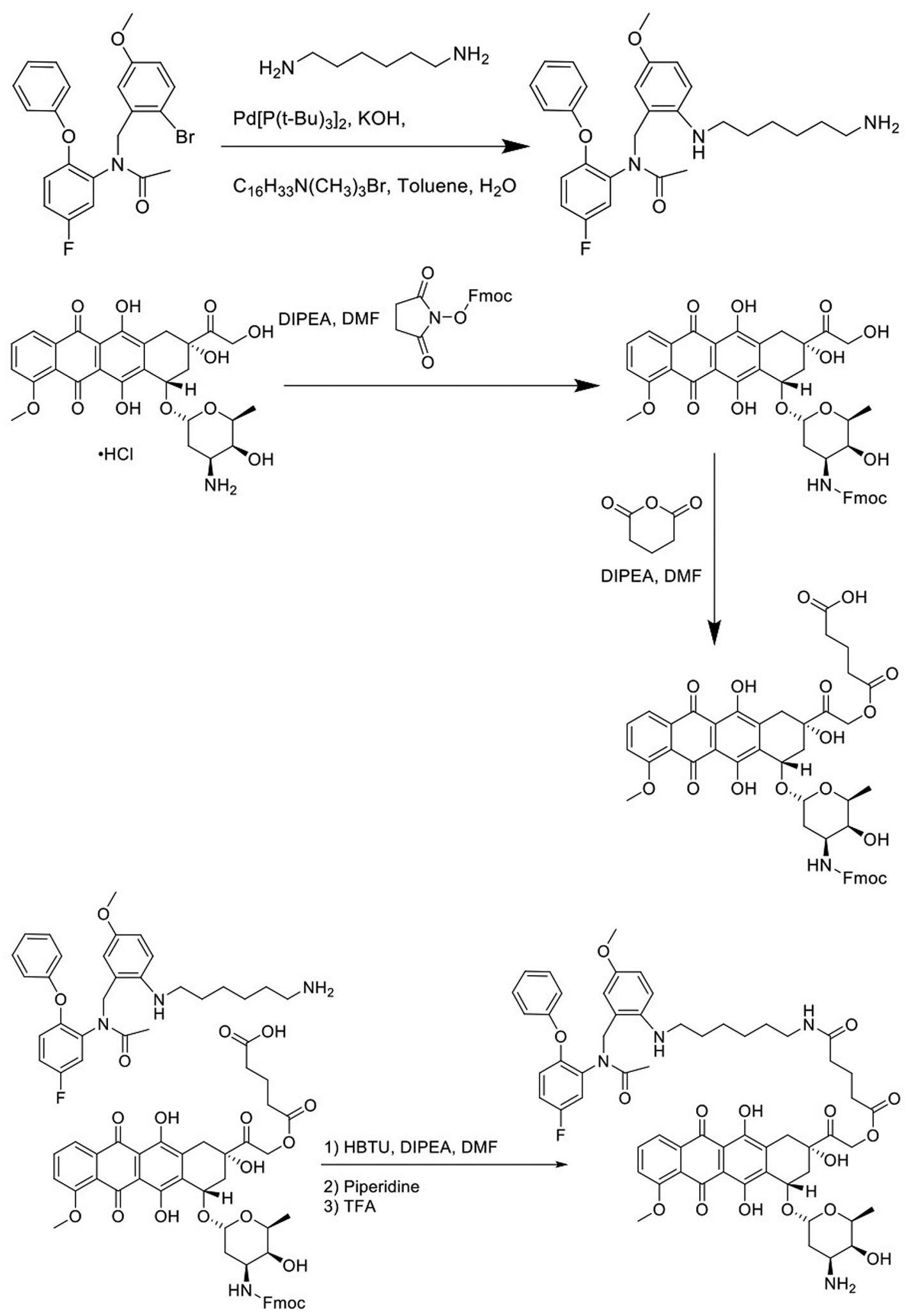

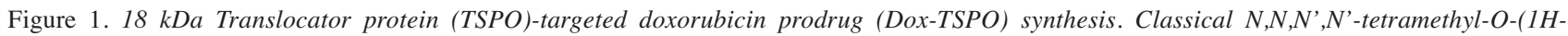
benzotriazol-1-yl) uranium hexafluorophosphate (HBTU) coupling of doxorubicin and the TSPO ligand followed by fluorenylmethoxycarbonyl protecting group (Fmoc) deprotection, with a yield of 82\%. DIPEA: N,N-Di-isopropylethylamine; DMF: dimethylformamide; TFA: trifluoroacetic acid. 


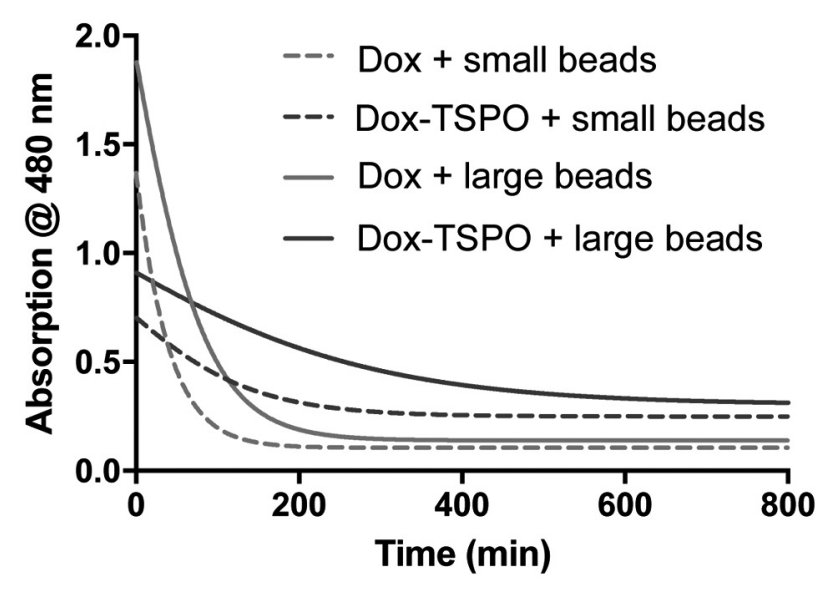

Figure 2. Comparison of loading efficacy of doxorubicin (Dox) and the $18 \mathrm{kDa}$ translocator protein (TSPO)-targeted doxorubicin prodrug (DoxTSPO) into 100-300 $\mu \mathrm{m}$ (small) and 300-500 $\mu \mathrm{m}$ (large) DC beads ${ }^{T M}$.

Cells. HT-29, and HCT-116 CRC cell lines and Hep G2 and Hep 3B hepatocellular carcinoma (HCC) cell lines were purchased from the American Type Culture Collection (ATCC; Manassas, VA, USA). The HT29 cells were maintained in McCoy's 5A medium with Lglutamine also purchased from the ATCC. The HCT-116, Hep 3B, and Hep G2 cells were maintained in DMEM from Sigma-Aldrich. Both media were supplemented with $10 \%$ fetal bovine serum (Sigma-Aldrich) and $100 \mathrm{U} / \mathrm{ml}$ penicillin. Cells were incubated at $37^{\circ} \mathrm{C}$ with $95 \%$ air and $5 \% \mathrm{CO}_{2}$.

In-vitro cell-viability assays. Stock solutions of 1-2.5 mM were made with doxorubicin and Dox-TSPO using DMSO as a solvent. Cells were cultured in $55 \mathrm{~cm}^{2}$ flasks. When the culture flasks were saturated, cells were resuspended using trypsin and seeded into 96well plates, $100 \mu \mathrm{l}$ per well of the cells in medium, yielding approximately $5.7 \times 10^{4}$ cells per well (assuming $1 \times 10^{5}$ cells $/ \mathrm{cm}^{2}$ in a $100 \%$ confluent culture). The plates were allowed to incubate for $24 \mathrm{~h}$. The medium was then removed and replaced with fresh medium with either 0,10 , or $50 \mu \mathrm{M}$ of Dox-TSPO or doxorubicin. Dilutions were performed immediately before the addition of the drugs to cells using distilled water. The number of viable cells was then measured at 24, 48, 60, and $72 \mathrm{~h}$ using the CellTiter-Glo ${ }^{\circledR}$ Luminescent Cell Viability Assay (Promega, Madison, MI, USA) according to the manufacturer's instructions. Luminescence of plates was read using a Synergy H4 Hybrid Multi-Mode Microplate Reader (BioTek, Winooski, VT, USA) 10 minutes after addition of the solution. Two independent experiments were performed in triplicate.

Statistical analysis. Data from cell viability studies are expressed as mean values with standard error (SE). Data were collated from two separate experiments performed in triplicate. Statistical comparisons of Dox-TSPO versus doxorubicin for each time point and drug concentration was performed using two-sided $t$-tests. All calculations were performed using PRISM 6 (GraphPad Software Inc., La Jolla, CA, USA). $p$-Values of less than 0.05 were considered statistically significant.

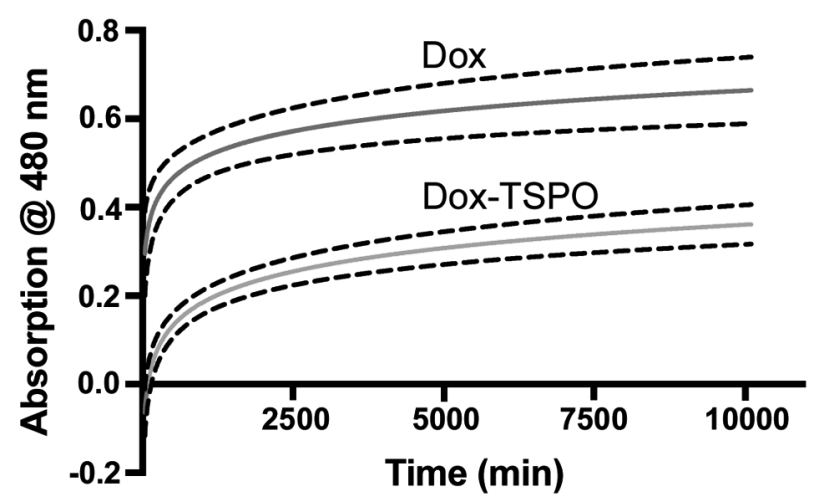

Figure 3. Comparison of elution kinetics of doxorubicin (Dox) and the $18 \mathrm{kDa}$ translocator protein (TSPO)-targeted doxorubicin prodrug (Dox-TSPO) from DC beads ${ }^{T M}$ in Dulbecco's minimum essential cell medium. Results are shown as the mean \pm standard error of independent trials using 100-300 $\mu \mathrm{m}$ and 300-500 $\mu \mathrm{m}$ DC beads ${ }^{T M}$.

\section{Results}

Prodrug synthesis. The prodrug was synthesized as per Figure $1 .{ }^{1} \mathrm{H}$ NMR, ${ }^{13} \mathrm{C}$ NMR and MS analysis confirmed correct chemical structure. For greater detail regarding prodrug synthesis and NMR spectra (please see the Supplementary Material).

Loading doxorubicin and Dox-TSPO into beads. Dox-TSPO readily loaded into $\mathrm{DC}$ beads ${ }^{\mathrm{TM}}$ in a fashion similar to doxorubicin (22). When DC beads ${ }^{\mathrm{TM}}$ were charged with the orange-colored Dox-TSPO solution, the color of the drug solution slowly diminished. Simultaneously, as the colored drug molecules moved inside the bead cavities, the bluecolored beads turned red. Eighty-eight percent of Dox-TSPO was loaded versus over $99 \%$ of the same amount of doxorubicin into a $200 \mu \mathrm{l}$ solution of DC beads ${ }^{\mathrm{TM}}$. Free doxorubicin required a 3 -h loading time and Dox-TSPO a 5$\mathrm{h}$ loading time in order to reach equilibrium (Figure 2). There was no significant difference in loading rates between the $100-300 \mu \mathrm{m}$ and $300-500 \mu \mathrm{m}$ DC beads ${ }^{\mathrm{TM}}$.

Release of doxorubicin and Dox-TSPO from beads. The majority of Dox-TSPO was released in the first 3 days from the DC beads ${ }^{\mathrm{TM}}$, after which the process became progressively slower as it approached equilibrium. DoxTSPO reached maximum release in 1 day and greater than $30 \%$ of the drug was eluted after 3 days (Figure 3 ). There were similar rates of release with the 100-300 $\mu \mathrm{m}$ and 300$500 \mu \mathrm{m}$ DC beads $^{\mathrm{TM}}$. Dox-TSPO was not completely released, and the beads did not lose the characteristic dark red color indicating the presence of residual Dox-TSPO at 
Table I. Comparison of loss of viable cells following incubation with the $18 \mathrm{kDa}$ translocator protein (TSPO)-targeted doxorubicin prodrug (DoxTSPO) versus doxorubicin at different time points and concentrations in colorectal cancer (HT-29 and HCT-116) and hepatocellular carcinoma (Hep G2 and Hep 3B) cell lines.

\begin{tabular}{|c|c|c|c|c|c|c|c|c|c|c|c|c|}
\hline \multirow[b]{3}{*}{ Timepoint } & \multicolumn{12}{|c|}{ Dox-TSPO $(\mu \mathrm{M})$} \\
\hline & \multicolumn{3}{|c|}{ HT-29 } & \multicolumn{3}{|c|}{ HCT-116 } & \multicolumn{3}{|c|}{ Hep G2 } & \multicolumn{3}{|c|}{ Hep 3B } \\
\hline & 10 & 25 & 50 & 10 & 25 & 50 & 10 & 25 & 50 & 10 & 25 & 50 \\
\hline $24 \mathrm{~h}$ & $*$ & $*$ & $*$ & & & & & $*$ & $*$ & $*$ & $*$ & $*$ \\
\hline $48 \mathrm{~h}$ & $*$ & & $*$ & $*$ & $*$ & $*$ & $*$ & $*$ & $*$ & & & \\
\hline $60 \mathrm{~h}$ & $*$ & & & $*$ & $*$ & $*$ & $*$ & $*$ & $*$ & $*$ & $*$ & $*$ \\
\hline $72 \mathrm{~h}$ & * & $*$ & $*$ & $*$ & $*$ & & & & & & & \\
\hline
\end{tabular}

*Significant reduction in number of viable cells when compared to free doxorubicin.

equilibrium. Doxorubicin was also partially retained in the beads due to its strong ionic interaction with the sulfates, however, it demonstrated a higher proportion of drug elution, with $50 \%$ of the drug having been released at 3 days $(23,24)$.

In vitro cell-viability assays. Table I and Figure 4 summarize the cell viability results of Dox-TSPO and free doxorubicin against HT-29, and HCT-116 CRC cell lines and the Hep G2 and Hep 3B HCC cell lines. The HT-29 cell line overexpresses TSPO as documented in the NCI-60 Cancer Microarray database. HCT-116 expresses the median amount of TSPO when compared to cancer cell lines in the NCI-60 database and Hep G2 and Hep 3B both underexpress TSPO (25). A significant time- and concentration-dependent reduction in viable cells in all cell lines with both Dox-TSPO and doxorubicin versus controls was observed $(p<0.05)$. In the HT-29, Hep G2, and Hep 3B cell lines, Dox-TSPO caused a significant loss of viable cells at earlier time points than did doxorubicin. In these three cell lines, there was a significant loss of viable cells when incubated with $10 \mu \mathrm{M}$ of Dox-TSPO at $24 \mathrm{~h}$, with doxorubicin not causing a significant loss until $48 \mathrm{~h}(p<0.05$ for all). No difference in onset of significant loss in number of viable cells was seen at the other concentrations. In the HCT-116 cell line, both Dox-TSPO and doxorubicin caused a significant reduction in number of viable cells at all concentrations at $24 \mathrm{~h}(p<0.05)$.

In the HT-29, HCT-116, and Hep G2 cell lines, at the majority of time and concentration points, Dox-TSPO performed superiorly to free doxorubicin (Table I). In the HCT-116 cell line at 48 and $72 \mathrm{~h}$ and in the HT-29 cell line at 24 and $72 \mathrm{~h}$, Dox-TSPO resulted in significantly higher loss of viable cells versus doxorubicin at all concentrations $(p<0.05$ for all). In HT-29 cells, $10 \mu \mathrm{M}$ of Dox-TSPO also led to significantly superior loss of viable cells compared to doxorubicin at 48 and $60 \mathrm{~h}$ and $50 \mu \mathrm{M}$ at $60 \mathrm{~h}(p<0.05)$. Of the cell lines with a low expression of TSPO, a consistent increased loss in viable cells with Dox-TSPO was also found at 24,48 , and $60 \mathrm{~h}$, with a plateau effect in the Hep G2 cell line at $72 \mathrm{~h}$ for all concentrations ( $p<0.05$ for all). A consistent difference in number of viable cells between the two drugs was not observed in the Hep 3B line.

When cell lines expressing TSPO were directly compared to those with low TSPO expression, they were found to be more sensitive to Dox-TSPO with 2- to 4- fold more viable cells in the doxorubicin-treated groups compared to the DoxTSPO-treated groups at $72 \mathrm{~h}$. There was not a significant difference in number of viable cells between the Dox-TSPOand doxorubicin-treated Hep G2 and Hep 3B cell lines at this time point.

\section{Discussion}

Dox-TSPO was successfully synthesized as confirmed by NMR and MS analysis. It readily loaded into DEB-TACE beads, albeit at a slower rate than free doxorubicin, 5 versus $3 \mathrm{~h}$. Doxorubicin and Dox-TSPO are hydrophobic molecules. When the molecules enter the DEB-TACE beads, water molecules are displaced. The larger Dox-TSPO requires more water molecules to be released from the bead cavity. This adds an energy burden, likely contributing to the longer drug loading time. In addition, the ionic interaction (22) between the protonated primary amine on doxorubicin and sulfonate groups inside the bead cavity is the main driving force when loading doxorubicin into DEB-TACE beads. The added secondary amine on the TSPO targeting moiety is a $p$ methoxyaniline derivative, which is not protonated at the $\mathrm{pH}$ level of the loading solution. Therefore, the loading process of Dox-TSPO did not kinetically benefit from the additional amine group, also likely contributing to the slower loading. Clinically, this difference in loading time may not be significant given beads are preloaded with chemotherapy prior to DEB-TACE procedures. 

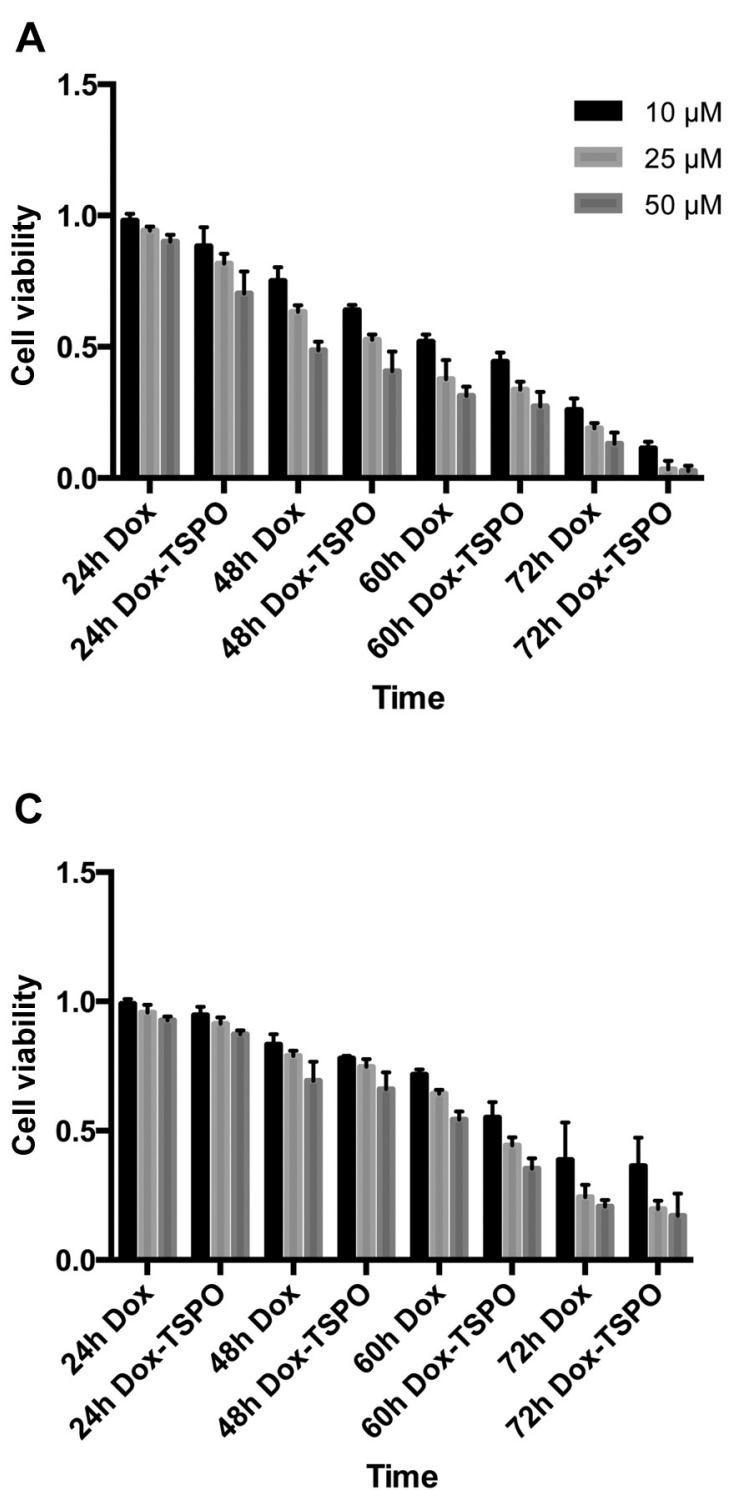

B

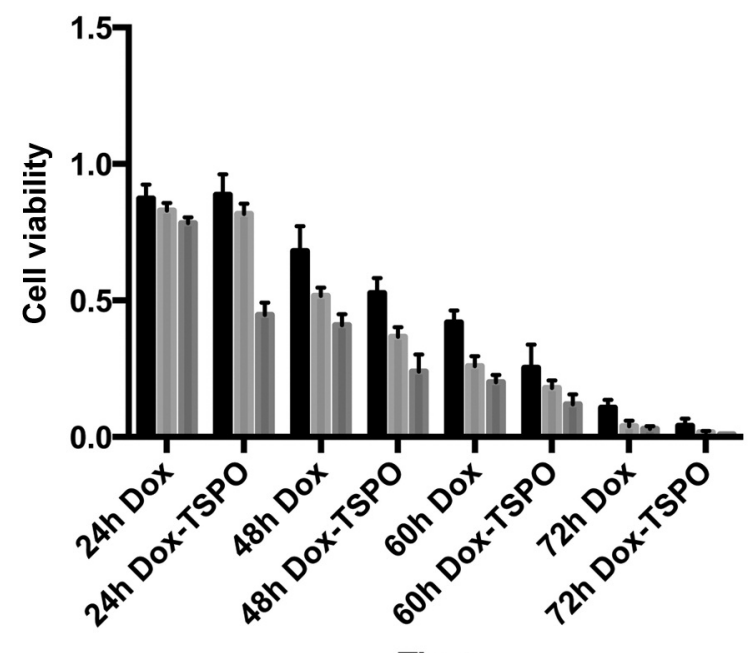

Time

D

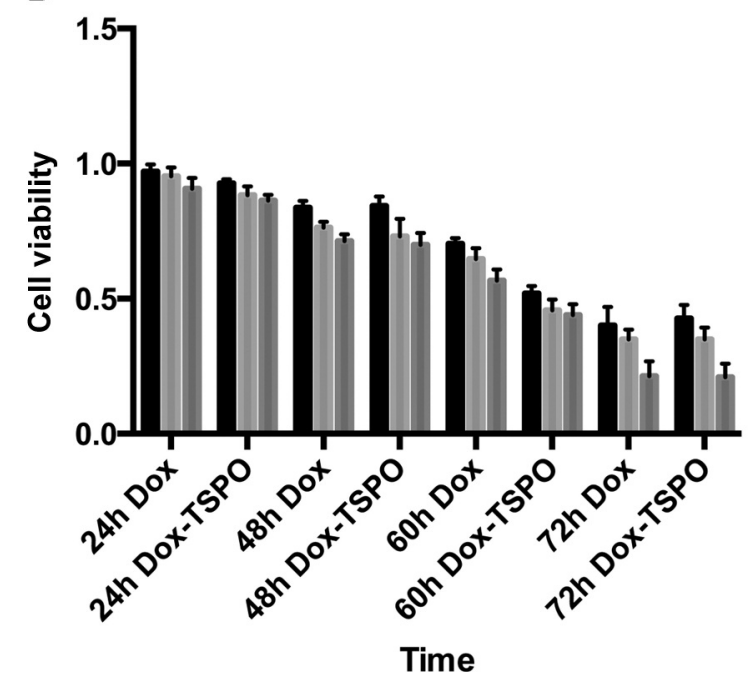

Figure 4. Cell viability of HT-29 (A) and HCT-116 (B) colorectal cancer cell lines and Hep G2 (C) and Hep 3B (D) hepatocellular carcinoma cell lines. The number of viable cells following incubation with the $18 \mathrm{kDa}$ translocator protein (TSPO)-targeted doxorubicin prodrug (Dox-TSPO) or free doxorubicin (Dox) is presented relative to those of the control (mean \pm standard error). Two independent trials were performed in triplicate.

After Dox-TSPO entered the bead cavity, sulfonate groups were expected to surround the molecule. In this significantly different solvation environment, the acid dissociation constant $\left(\mathrm{K}_{\mathrm{a}}\right)$ of the secondary amine may have shifted (23), resulting in protonation and therefore additional ionic interactions with nearby sulfonates. The thermodynamically favored interaction was demonstrated when attempts to release the molecule from the beads using saline solution were found to be unsuccessful. Unlike doxorubicin, which was promptly released from DEB-TACE bead in saline, little to no Dox-TSPO was released under the same condition. The ionic strength of the saline solution was insufficient to overcome the fortified force and displace the Dox-TSPO from inside beads. We later found that DMEM is capable of releasing Dox-TSPO from the DC beads ${ }^{\mathrm{TM}}$, albeit more slowly when compared to free doxorubicin. This slower elution may actually be of benefit in cancer treatment as this can allow for longer exposure times of tumor tissues to the chemotherapeutic agent.

We hypothesized that Dox-TSPO would be more effective in causing a loss of viability in cell lines with high TSPO expression secondary to cell targeting and enhanced uptake. 
This was supported by our data as our two cell lines that expressed TSPO showed increased sensitivity to Dox-TSPO when compared to free doxorubicin, with the doxorubicintreated cells having a 2- to 4-fold higher number of viable cells following incubation for $72 \mathrm{~h}$. The cell lines that underexpress TSPO did not show a significant difference in viable cells following this length of incubation. This finding suggests specificity of the prodrug.

Loss of viable HT-29, Hep G2, and Hep 3B cells occurred at a significantly faster rate with Dox-TSPO than with free doxorubicin at identical concentrations. It is unclear whether the same would hold true for the HCT-116 cell line as both agents caused significant loss of viable cells at the first time point. This is likely explained by the targeting moiety accelerating drug uptake into cells. A similar study using CD19-targeted doxorubicin in Burkitt's lymphoma cells showed increased efficacy and specificity of the targeted drug when compared to free doxorubicin alone. Subsequent flow cytometry demonstrated that the targeted drug accumulated more rapidly within the cells, thus explaining its enhanced effect (26). Future internalization studies would be important to perform to confirm this mechanism for DoxTSPO. The presumed accelerated uptake of Dox-TSPO, is a benefit as it can reduce the time that doxorubicin stays in the systemic circulation.

Further investigation needs to be performed to elucidate the mechanism of action of Dox-TSPO, its in vivo effects, localization, efficacy, and adverse effects. If further studies continue to support the improved targeting of doxorubicin to tumor cells using TSPO as a ligand, there is potential for the ligand to be conjugated with other chemotherapeutic agents to expand its utility.

We were able to successfully synthesize, load, and release our novel mitochondria-targeted doxorubicin prodrug from DC beads ${ }^{\mathrm{TM}}$. In addition, the prodrug resulted in superior loss of viable cells when compared to doxorubicin in human HCT-116 and HT-29 CRC cancer cell lines and similar rates of loss of viable cells to doxorubicin in the human Hep G2 and Hep 3B HCC cell lines. Because of its equivalent to superior effectiveness in reducing viable cells when compared to free doxorubicin and its capacity to be used with DEB-TACE beads, the targeted prodrug is a promising candidate for targeted and directed cancer treatment in CRC.

\section{Conflicts of Interest}

The Authors have no relevant conflicts of interest.

\section{Authors' Contributions}

Study Conception and study design: HSK and MB. Collected the data: JBJ, XL, MX and JML. Contributed data and analysis tools: JBJ, XL, MX and JML. Performed analysis: JBJ, XL, MX and JML.
Initial draft of the article: JBJ and XL. Editing: JBJ, XL, MX, JML, MH and HSK. Final approval: JBJ, XL, MX, JML, MH and HSK. Supervision of the study: HSK and MH.

\section{Supplementary Material}

Available at: https://sites.google.com/view/dox-tspo-supplementalmaterial/home

\section{References}

1 Jemal A, Siegel R, Ward E, Hao Y, Xu J, Murray T and Thun M: Cancer statistics 2008. CA Cancer J Clin 58(2): 71-96, 2008. PMID: 18287387. DOI: 10.3322/CA.2007.0010

2 Leporrier J, Maurel J, Chiche L, Bara S, Segol P and Launoy G: A population-based study of the incidence, management and prognosis of hepatic metastases from colorectal cancer. Br J Surg 93(4): 465-474, 2006. PMID: 16523446. DOI: 10.1002/bjs.5278

3 Faivre J, Manfredi S and Bouvier AM: [Epidemiology of colorectal cancer liver metastases]. Bull Acad Natl Med 187(5): 815-822; discussion 22-23, 2003. PMID: 14979048.

4 Tellez C, Benson AB 3rd, Lyster MT, Talamonti M, Shaw J, Braun MA, Namcek AA and Vogelzing RL: Phase II trial of chemoembolization for the treatment of metastatic colorectal carcinoma to the liver and review of the literature. Cancer 82(7): 1250-1259, 1998. PMID: 9529016. DOI: 10.1002/(sici)10970142(19980401)82:7<1250::aid-cncr7>3.0.co;2-j

5 Fiorentini G, Aliberti C, Tilli M, Mulazzani L, Graziano F, Giordani P, Mambrini A, Montagnani F, Alessandroni R, Catalano V and Coschiera P: Intra-arterial infusion of irinotecanloaded drug-eluting beads (DEBIRI) versus intravenous therapy (FOLFIRI) for hepatic metastases from colorectal cancer: Final results of a phase III study. Anticancer Res 32(4): 1387-1395, 2012. PMID: 22493375.

6 Austin CJ, Kahlert J, Kassiou M and Rendina LM: The translocator protein (TSPO): A novel target for cancer chemotherapy. Int J Biochem Cell Biol 45(7): 1212-1216, 2013. PMID: 23518318. DOI: 10.1016/j.biocel.2013.03.004

7 Papadopoulos V, Amri H, Boujrad N, Cascio C, Culty M, Garnier M, Hardwisk M, Li H, Vidic B, Brown AS, Reversa JL, Bernassau $\mathrm{JM}$ and Drieu K: Peripheral benzodiazepine receptor in cholesterol transport and steroidogenesis. Steroids 62(1): 21-28, 1997. PMID: 9029710. DOI: 10.1016/s0039-128x(96)00154-7

8 Katz Y, Eitan A, Amiri Z and Gavish M: Dramatic increase in peripheral benzodiazepine binding sites in human colonic adenocarcinoma as compared to normal colon. Eur J Pharmacol 148(3): 483-484, 1988. PMID: 2838309. DOI: $10.1016 / 0014-$ 2999(88)90135-5

9 Katz Y, Ben-Baruch G, Kloog Y, Menczer J and Gavish M: Increased density of peripheral benzodiazepine-binding sites in ovarian carcinomas as compared with benign ovarian tumours and normal ovaries. Clin Sci 78(2): 155-158, 1990. PMID: 2155741. DOI: $10.1042 / \mathrm{cs} 0780155$

10 Benavides J, Cornu P, Dennis T, Dubois A, Hauw JJ, MacKenzie ET, Sazdovitch V and Scatton B: Imaging of human brain lesions with an omega 3 site radioligand. Ann Neurol 24(6): 708-712, 1988. PMID: 2849920. DOI: 10.1002/ana.410240603

11 Miettinen H, Kononen J, Haapasalo H, Helen P, Sallinen P, Harjuntausta T, Helin $\mathrm{H}$ and Alho $\mathrm{H}$ : Expression of peripheral- 
type benzodiazepine receptor and diazepam binding inhibitor in human astrocytomas: Relationship to cell proliferation. Cancer Res 55(12): 2691-2695, 1995. PMID: 7780986.

12 Han Z, Slack RS, Li W and Papadopoulos V: Expression of peripheral benzodiazepine receptor (PBR) in human tumors: Relationship to breast, colorectal, and prostate tumor progression. J Recept Signal Transduct Res 23(2-3): 225-238, 2003. PMID: 14626449 DOI: $10.1081 /$ rrs-120025210

13 Nagler R, Ben-Izhak O, Savulescu D, Krayzler E, Akrish S, Leschiner S, Otradnov I, Zeno S, Veenman L and Gavish M: Oral cancer, cigarette smoke and mitochondrial $18 \mathrm{kDa}$ translocator protein (TSPO) - In vitro, in vivo, salivary analysis. Biochim Biophys Acta 1802(5): 454-461, 2010. PMID: 20085808. DOI: $10.1016 /$ j.bbadis.2010.01.008

14 Samuelson LE, Dukes MJ, Hunt CR, Casey JD and Bornhop DJ: TSPO targeted dendrimer imaging agent: synthesis, characterization, and cellular internalization. Bioconjug Chem 20(11): 2082-2089, 2009. PMID: 19863077. DOI: 10.1021/bc9002053

15 Wyatt SK, Manning HC, Bai M, Bailey SN, Gallant P, Ma G, Mclntosh L and Bornhop DJ: Molecular imaging of the translocator protein (TSPO) in a pre-clinical model of breast cancer. Mol Imaging Biol 12(3): 349-358, 2010. PMID: 19949989. DOI: 10.1007/s11307-009-0270-8

16 Margiotta N, Denora N, Ostuni R, Laquintana V, Anderson A, Johnson SW, Trapani G and Natile G: Platinum(II) complexes with bioactive carrier ligands having high affinity for the translocator protein. J Med Chem 53(14): 5144-5154, 2010. PMID: 20568783. DOI: 10.1021/jm100429r

17 Zhang S, Yang L, Ling X, Shao P, Wang X, Edwards WB and Bai M: Tumor mitochondria-targeted photodynamic therapy with a translocator protein (TSPO)-specific photosensitizer. Acta Biomater 28: 160-170, 2015. PMID: 26432436. DOI: 10.1016/ j.actbio.2015.09.033

18 Kalva SP, Iqbal SI, Yeddula K, Blaszkowsky LS, Akbar A, Wicky $\mathrm{S}$ and Zhu AX: Transarterial chemoembolization with doxorubicineluting microspheres for inoperable hepatocellular carcinoma. Gastrointest Cancer Res 4(1): 2-8, 2011. PMID: 21464864.

19 Booser DJ and Hortobagyi GN: Anthracycline antibiotics in cancer therapy. Focus on drug resistance. Drugs 47(2): 223-258, 1994. DOI: $10.2165 / 00003495-199447020-00002$

20 Bai M, Rone MB, Papadopoulos V and Bornhop DJ: A novel functional translocator protein ligand for cancer imaging. Bioconjug Chem 18(6): 2018-2023, 2007. PMID: 17979225. DOI: $10.1021 / \mathrm{bc} 700251 \mathrm{e}$
21 Nagy A, Schally AV, Armatis P, Szepeshazi K, Halmos G, Kovacs M, Zarandi M, Groot K, Miyazaki M, Jungwirth A and Horvath J: Cytotoxic analogs of luteinizing hormone-releasing hormone containing doxorubicin or 2-pyrrolinodoxorubicin, a derivative 500-1,000 times more potent. Proc Natl Acad Sci USA 93(14): 7269-7273, 1996. PMID: 8692981. DOI: 10.1073/pnas.93.14.7269

22 Lewis AL, Gonzalez MV, Leppard SW, Brown JE, Stratford PW, Phillips GJ and Lloyd AW: Doxorubicin eluting beads-1: Effects of drug loading on bead characteristics and drug distribution. J Mater Sci Mater Med 18(9): 1691-1699, 2007. PMID: 17653626. DOI: 10.1007/s10856-006-0040-y

23 Urry DW, Gowda DC, Peng SQ, Parker TM and Harris RD: Design at nanometric dimensions to enhance hydrophobicityinduced $\mathrm{pK}_{\mathrm{a}}$ shifts. J Am Chem Soc 114(22): 8716-8717, 1992. DOI: $10.1021 / \mathrm{ja} 00048 \mathrm{a} 064$

24 Jordan O, Denys A, De Baere T, Boulens N and Doelker E: Comparative study of chemoembolization loadable beads: In vitro drug release and physical properties of dc bead and hepasphere loaded with doxorubicin and irinotecan. J Vasc Interv Radiol 21(7): 1084-1090, 2010. PMID: 20610183. DOI: 10.1016/j.jvir.2010.02.042

25 Scherf U, Ross DT, Waltham M, Smith LH, Lee JK, Tanabe L, Kohn KW, Reinhold WC, Myers TG, Andrews DT, Scudiero DA, Eisen MB, Sausville EA, Pommier Y, Botstein D, Brown $\mathrm{PO}$ and Weinstein JN: A gene expression database for the molecular pharmacology of cancer. Nat Genet 24(3): 236-244, 2000. PMID: 10700175. DOI: 10.1038/73439

26 de Menezes DEL, Kirchmeier MJ, Gagne JF, Pilarski LM and Allen TM: Cellular trafficking and cytotoxicity of anti-CD19targeted liposomal doxorubicin in B lymphoma cells. J Liposome Res 9(2): 199-228, 1999. DOI: 10.3109/08982109909024786

Received July 30, 2020

Revised August 16, 2020

Accepted August 23, 2020 